\title{
Effects of age, temperature, and disease on the refractoriness of human nerve and muscle ${ }^{1}$
}

\author{
J. D E L B E K E, J . K O P E C, A N D A. J . M C COMAS ${ }^{2}$ \\ From the MRC Group in Developmental Neurobiology and Department of Medicine (Neurology), \\ McMaster University, Hamilton, Ontario, Canada
}

SUMMARY Absolutely and relatively refractory periods have been investigated in terminal median nerve axons and in thenar muscle fibres in a variety of pathophysiological conditions. Marked increases in refractoriness were produced by cooling to $20^{\circ} \mathrm{C}$. Nerve fibre refractoriness was more pronounced in older subjects and diminished in myotonic dystrophy. In contrast, no significant changes in muscle fibre excitability could be demonstrated in either condition or in patients with renal failure or motor neuropathies.

A new technique was described by Kopec et al. (1978) for studying the absolutely ref ractory periods (ARP) and relatively refractory periods (RRP) of nerve and muscle fibres in man. The technique involved stimulating median motor nerve fibres electrically and recording the responses evoked in the thenar muscles with surface electrodes. Under normal circumstances, when a testing stimulus is given within a few milliseconds of a conditioning shock, the two muscle responses overlap in time and cannot be analysed separately in a satisfactory way. However, the response to a solitary stimulus should be the same as that to the conditioning shock; therefore, by subtracting the former from the responses to paired (conditioning and test) stimuli, the response to the testing shock alone can be seen. The necessary subtractions are carried out using a signal analyser in tandem with a programmable calculator and $\mathrm{X}-\mathrm{Y}$ plotter.

The purpose of the present investigation was to assess the effects of certain physiological and pathological variables upon refractoriness. The physiological variables were age and temperature; the clinical conditions chosen for study were a 'myopathy' (myotonic dystrophy), a metabolic disorder (chronic renal failure), and two motor neuropathies (amyotrophic lateral sclerosis and

\footnotetext{
'Presented in part at the Ninth International Congress of Electroencephalography and Clinical Neurophysiology, Amsterdam, September 1977.

${ }^{2}$ Address for correspondence and reprint requests Dr Alan McComas, Room 4U7, McMaster University Medical Centre, 1200 Main Street West, Hamilton, Ontario, Canada L8S $4 J 9$.

Accepted 9 August 1977
}

spinal muscular atrophy). It will be shown that, while refractoriness was found to be influenced profoundly by temperature, only slight changes could be demonstrated as consequences of aging or disease.

\section{Subjects and methods}

The experiments were performed with the approval of the Medical Ethics Committee of McMaster University, and informed consent was obtained from the subjects.

Aging Eighteen hands were examined in nine control subjects free of neuromuscular disease and aged between 40 and 68 years (mean age: $47.9 \pm 9.3 \mathrm{yr}$ ).

Temperature Ten experiments were performed on two male subjects, aged 28 and 48 years respectively.

Myotonic dystrophy Five patients of both sexes, aged between 15 and 46 years, were studied. All had moderate wasting and weakness of distal muscles together with clinical and EMG evidence of myotonia. One additional patient with myotonia congenita was also investigated.

Renal failure Four patients, all female and aged between 46 and 52 years, were studied. All were receiving haemodialysis two to three times a week. Motor neuropathy Three hands were studied in two female patients, aged 28 and 43 years respectively, with amyotrophic lateral sclerosis. Both had moderately severe generalised muscle weakness. A third patient, a male aged 47 years, had spinal muscular atrophy with a limb girdle distri- 
bution of weakness and pseudohypertrophy of calf and thigh muscles.

TEMPERATURE CONTROL

Except in the temperature study (see below) the temperature of the skin overlying the thenar muscles was maintained between $33^{\circ} \mathrm{C}$ and $35^{\circ} \mathrm{C}$. In the temperature study a temperature-monitoring probe was inserted subcutaneously so as to lie on the surface of the thenar muscles. In this last study the hand, with electrodes attached, was inserted into a thin rubber glove and then immersed in a waterbath.

\section{SUBTRACTION TECHNIQUE}

The technique for measuring refractoriness was that described by Kopec et al. (1978); the same use has been made of the terms $S_{2}, S_{3}$, for stimulation and $R_{2}, R_{3}$, for recording procedures.

\section{Results}

EFFECTS OF TEMPERATURE

Figure 1 shows the most complete set of results obtained in a series of 10 experiments. The values shown are the amplitudes of the thenar muscle responses to testing $\left(\mathbf{S}_{3}\right)$ stimuli delivered to the median nerve at the wrist at different times after conditioning $\left(\mathrm{S}_{2}\right)$ shocks; measurements of terminal motor latency have also been included. Four sets of curves are displayed, corresponding to thenar muscle temperatures of $20^{\circ}, 28^{\circ}, 34^{\circ}$, and $40^{\circ} \mathrm{C}$ respectively. Whereas no clear difference could be detected between the responses at the two higher temperatures $\left(34^{\circ}\right.$ and $\left.40^{\circ} \mathrm{C}\right)$, cooling the tissues to $28^{\circ}$ and $20^{\circ} \mathrm{C}$ produced a number of profound effects. Firstly, there was an increase in terminal motor latency (from $2.8 \mathrm{~ms}$ at $34^{\circ} \mathrm{C}$ to $4.8 \mathrm{~ms}$ at $20^{\circ} \mathrm{C}$ ), indicating that impulse conduction had been slowed in motor nerve fibres. Secondly, the degree of impulse slowing during the RRP of the nerve fibres was greatly increased this was shown by the extent of the change in terminal motor latency as the $S_{2} S_{3}$ interval was lengthened. At $34^{\circ} \mathrm{C}$ the change in latency was $1.2 \mathrm{~ms}$ while at the lower temperature it had extended to $4.0 \mathrm{~ms}$. That the ARPs of the muscle fibres were increased to rather greater extents than those of the nerve fibres is suggested by the shapes of the recovery curves. At the lower temperatures $\left(20^{\circ} \mathrm{C}\right.$ and $\left.28^{\circ} \mathrm{C}\right)$ the initial segments of the $\mathrm{S}$ curves are prolonged because of the presence of endplate potentials which could not give rise to propagated action potentials. Only when the $\mathrm{S}$. $\mathrm{S}$. interval was lengthened to $3 \mathrm{~ms}$ was it possible for some of the muscle fibres to fire impulses. On the basis of the endplate potentials, the shortest ARPO of the nerve fibres was estimated to be $1.8 \mathrm{~ms}$ aा⿱ $34^{\circ} \mathrm{C}$ and $40^{\circ} \mathrm{C}, 2.9 \mathrm{~ms}$ at $28^{\circ} \mathrm{C}$, and $5.5 \mathrm{~ms}$ a $20^{\circ} \mathrm{C}$ (Fig. 2); these estimates depended on the calculation of $T^{\prime}$ as described by Kopec et al. (1978). The ARPs of the muscle fibres could alsof be derived from measurements of $T^{\prime}$; the relativels large increase brought about by cooling is showro. in Fig. 3. When $T^{\prime}$ was estimated for $50 \%$ recovery? of the evoked muscle responses, the values were

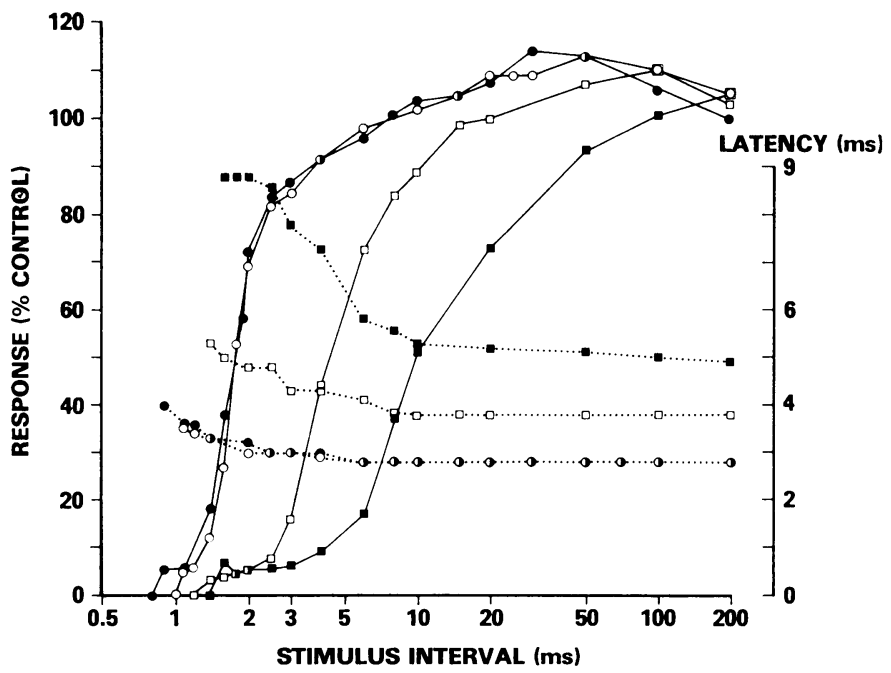

Fig. 1 Amplitudes (- - ) and terminal latencies ( - . - ) of thenar $R_{3}$ muscle responses to testing stimuli at $40^{\circ} \mathrm{C}(\bullet)$, $34^{\circ} \mathrm{C}(\curvearrowleft), 28^{\circ} \mathrm{C}(\square)$, and $20^{\circ} \mathrm{C}(\square)$. 
$2.3,4.5$, and $9.3 \mathrm{~ms}$ for temperatures of $34^{\circ}, 28^{\circ}$, and $20^{\circ} \mathrm{C}$ respectively (Fig. 2).

EFFECTS OF AGE

To investigate the effects of age, 18 hands were examined in nine control subjects aged between 40 and 68 years (mean age $47.9 \mathrm{yr}$ ), and the results were compared with those obtained by Kopec et

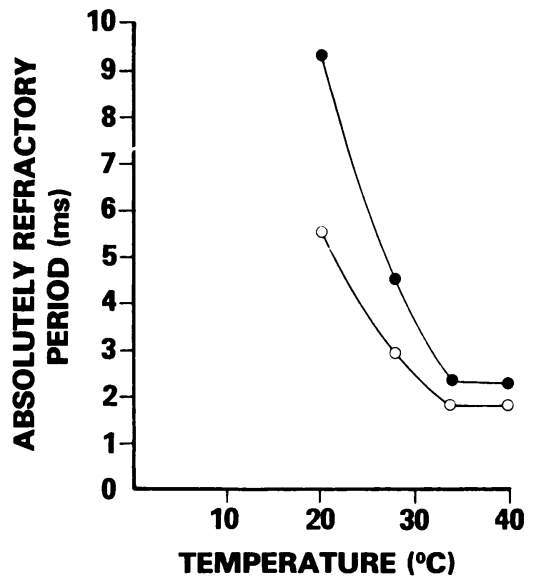

Fig. 2 Shortest ARPs of motor nerve terminals (c) and approximate mean ARPs of muscle fibres (•) at different temperatures.

al. (1978) in younger adults (19-29 years). Figure 4 shows the recovery of excitability in median nerve and thenar muscle fibres of the older subjects at different conditioning-test stimulus intervals. As the interval was lengthened the evoked muscle response enlarged, the resulting curve assuming the $S$ shape characteristic of the results in younger subjects. However, it can be seen that the recovery curve in the older subjects is displaced slightly to the right, indicating that comparatively fewer muscle fibres are responding at stimulus intervals of 1.4 to $4 \mathrm{~ms}$. The difference between the two curves is most marked at intervals of $1.8,2.0$ and $3 \mathrm{~ms}$ and is statistically significant $(\mathrm{P}<0.005)$. As in the younger subjects, the muscle responses to the testing stimuli in the older group exceeded the control values in amplitude at $10-100 \mathrm{~ms}$ intervals. The terminal motor latencies of the test responses $\left(R_{3}\right)$ are also displayed in Fig. 4, and it can be seen that similar prolongations at short conditioning-test intervals occurred in the younger and older subjects. As the stimulus interval was lengthened the terminal latency declined in both populations of subjects, remaining rather larger in older than in younger persons though not significantly so $(\mathrm{P}>0.1)$.

Figure 6 shows the recovery of muscle excitability in terms of the time elapsing between the onsets of the conditioning and test responses $\left(R_{2}\right.$ and $\mathrm{R}_{3}$ respectively); this time corresponds to $T^{\prime}$ as determined by Kopec et al. (1978). As discussed by these authors, this time can be used in the calculation of muscle fibre ARPs. It can be seen that this excitability recovery curve, unlike that determined for stimulus intervals, shows no obvious difference when plotted for older and younger subjects.

\section{EFFECTS OF DISEASE}

Thirteen experiments were conducted on patients with a variety of neuromuscular diseases.

Myotonic dystrophy Five patients were investigated with myotonic dystrophy. The averaged excitability curve, when plotted for conditioningtest stimulus intervals (Fig. 5), showed a recovery which was faster than that in controls at intervals of 1.2 and $1.6 \mathrm{~ms}(\mathrm{P}<0.001)$. When excitability was measured in terms of response interval $\left(T^{\prime}\right)$, however, the results for the dystrophic patients were very similar to the values in controls (Fig. 6). Figure 5 shows that there were no significant differences from normal in terminal motor latency as the stimulus interval was lengthened. The onc patient investigated with myotonia congenita gave results which were within the normal range.

Motor neuropathy Three hands were studied in two women, aged 28 and 43 years respectively, with motoneurone disease. A further hand was investigated in a 47 year old man with spinal muscular atrophy; on both clinical and electrophysiological grounds the disease appeared to be limited to the limb girdle and trunk musculature. In these patients the recovery of excitability, plotted as a function either of stimulus interval or of response interval, was not significantly different from that of a control population of 22 subjects with a similar mean age (Fig. 6).

Renal failure Four female patients aged 46 to 52 years with renal failure were studied; all required regular dialysis two to three times a week and showed evidence of mild neuropathic involvement of the intrinsic muscles of the hands. When expressed in terms of response intervals, the excitability recovery curves were normal (Fig. 6).

\section{Discussion}

The aim of the present study has been to explore membrane function in human nerve and muscle 

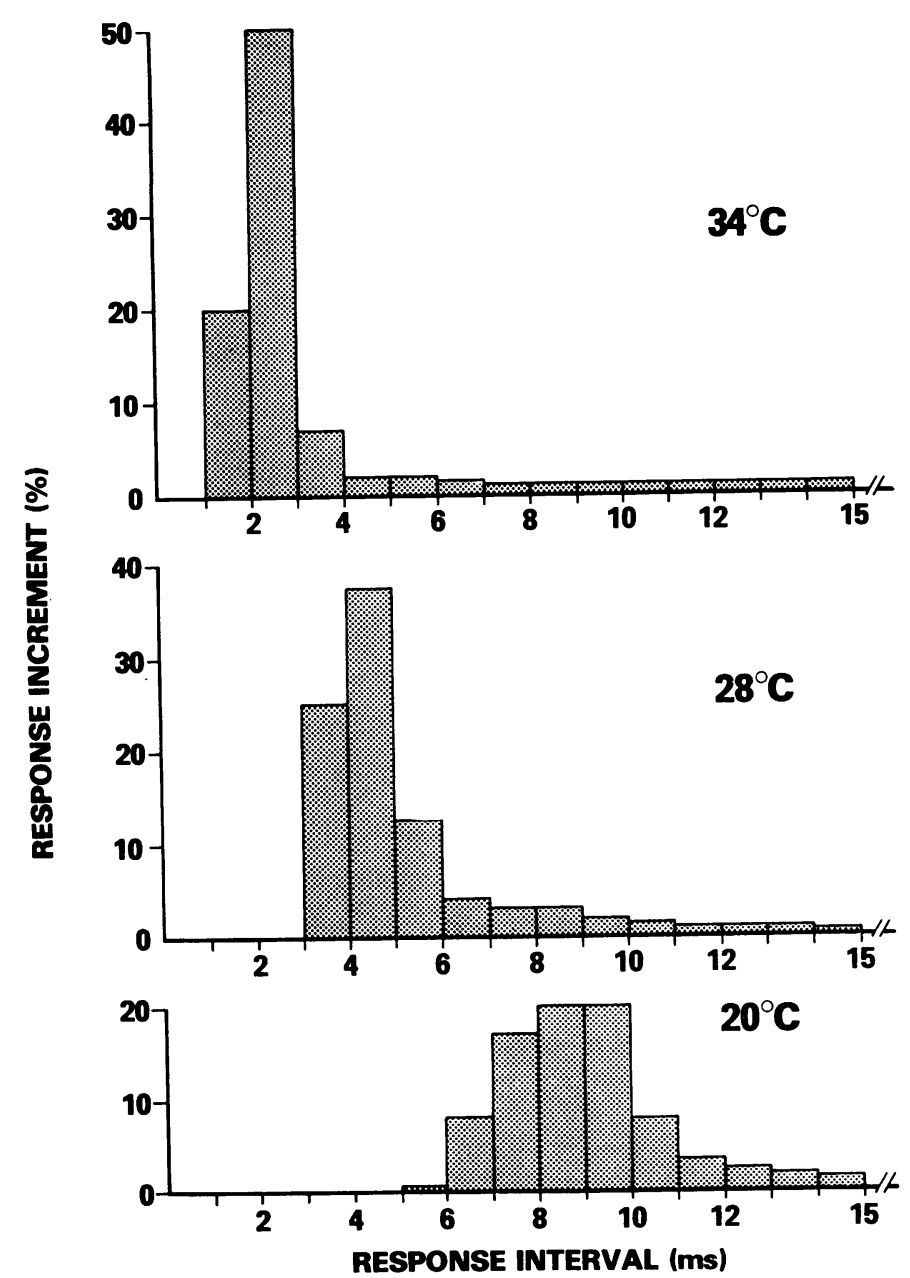

Fig. 3 Increase in amplitude of $R_{3}$ muscle response as a function of response interval at different temperatures. These two functions approximate to the number of muscle fibres and their ARPS respectively. fibres in different physiological and pathological states. The membrane property chosen for examination has been the recovery of excitability after the initiation and propagation of an action potential. From the voltage-clamp experiments of Hodgkin and Huxley (1952), it is known that further excitation will be possible only if an adequate number of sodium channels in the membrane are available to be switched open. The temporary inactivation of these channels at the end of the preceding impulse is partly responsible for the phenomenon of refractoriness; the other factor is a transient rise in potassium permeability. If the number of sodium channels in the membrane is reduced or if the channels have been inactivated (as by tetrodotoxin or local anaes- thetics) refractoriness will be increased. It was thought possible that a reduction in the number of channels might occur in otherwise normal nerve and muscle fibre membranes as a consequence of aging. A similar reduction might also be anticipated in muscular dystrophy for there is now a growing body of evidence for the presence of a membrane defect in each of the main types (for review, see Rowland, 1976). In electrolyte disorders, such as those accompanying renal failure, a fall in the resting membrane potential might cause a continuing inactivation of sodium channels, even though a full complement might be present in the membrane (accommodation phenomenon).

Following the reasoning above, it was 


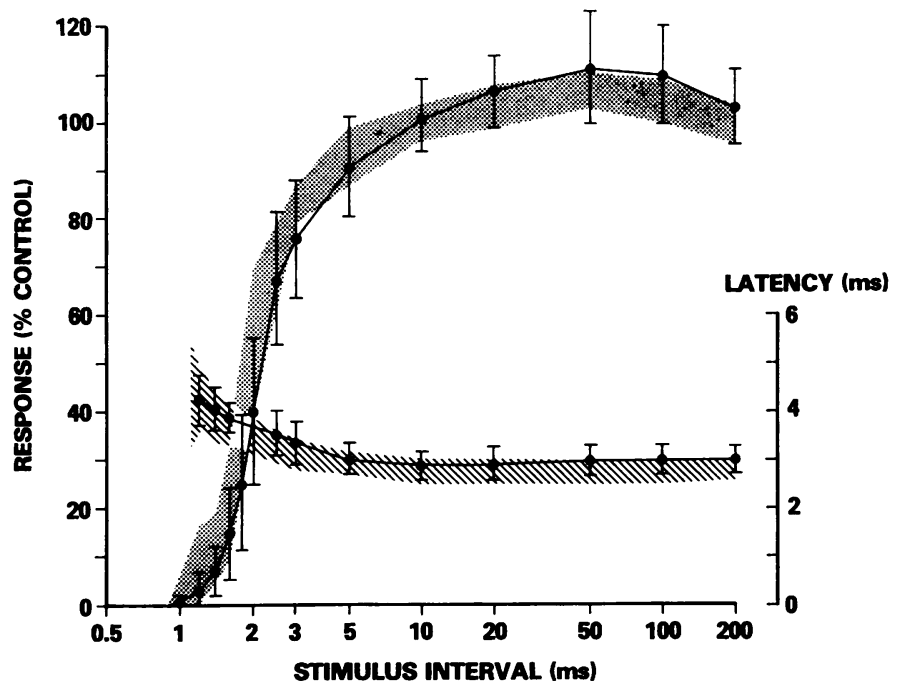

Fig. 4 Mean amplitudes and terminal latencies of $R_{3}$ responses at different conditioning-test stimulus intervals in older control population. The stippled and hatched areas show the corresponding mean values $\pm 1 S D$ for younger controls; see text.

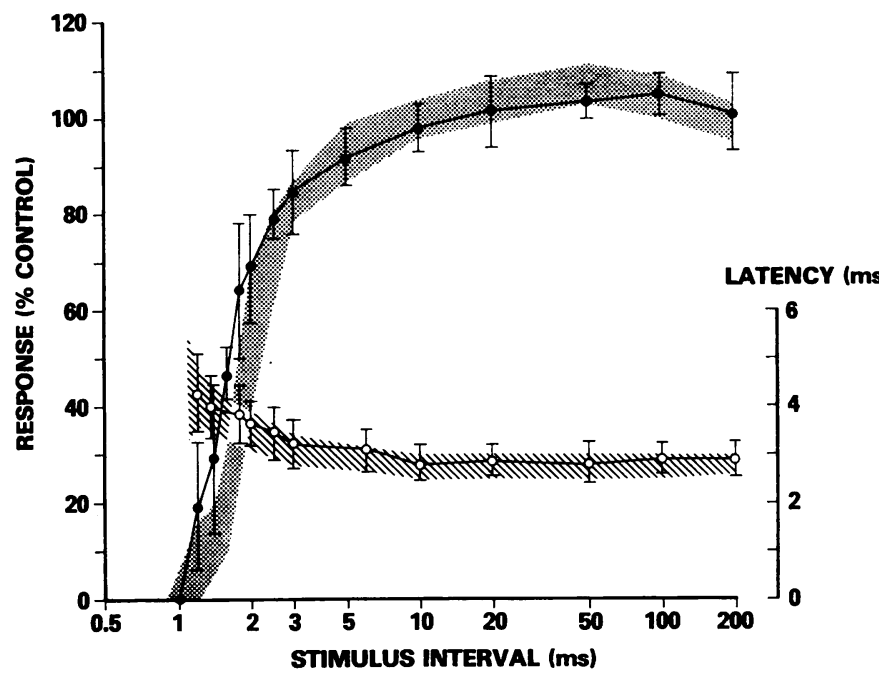

Fig. 5 Mean amplitudes and terminal latencies of $R_{3}$ responses at different conditioning-test stimulus intervals in five patients with myotonic dystrophy. The stippled and hatched areas show the corresponding mean values $\pm 1 S D$ for controls matched approximately for age.

hoped that abnormal refractoriness might be demonstrated in a variety of pathophysiological conditions. The most striking abnormalities which we have been able to demonstrate were those associated with cooling. It is an old observation that cooling increases refractoriness, and it is now known that this effect results from prolongation of the periods of sodium channel inactivation and of potassium channel opening. Most experiments have been conducted on nerve, however, and we are unaware of other investigations in man. In frog sartorius muscle fibres, Buchthal and Engbaek (1963) found that the ARP was increased by cooling from $25^{\circ} \mathrm{C}$ to $14^{\circ} \mathrm{C}$ with a $\mathrm{Q}_{10}$ of 2.0 (or rather 0.5 , taking into account the negative relationship between temperature and refractory period). In the present study the relationship between temperature and refractoriness was found to be distinctly non-linear; while no significant difference in refractoriness could be demonstrated between $34^{\circ} \mathrm{C}$ and $40^{\circ} \mathrm{C}$, marked increases in ARP and RRP were found at lower temperatures. 


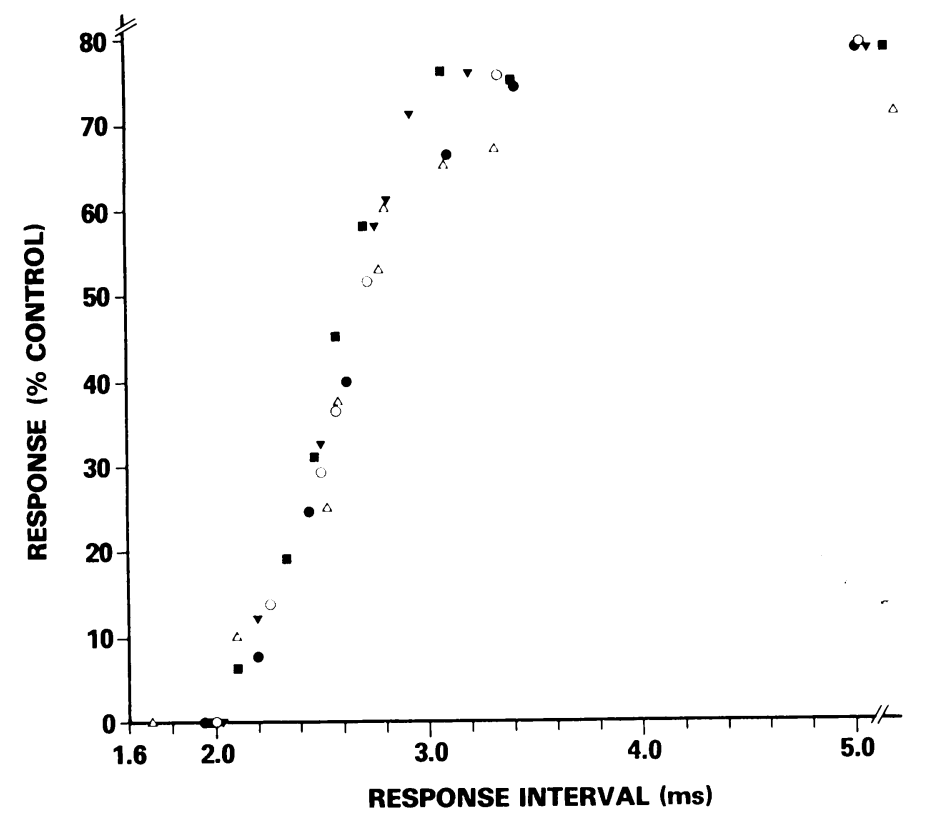

Fig. 6 Measurements of $R_{3}$ response area (voltage $\times$ time) at different response intervals in 'young' controls ( ). older controls (O), and in patients with motor neuropathy $(\boldsymbol{\square})$, renal failure $(\boldsymbol{\nabla})$, and myotonic dystrophy $(\triangle)$.

As Kopec et al. (1978) have pointed out, there is often difficulty in individual experiments of this type in deciding whether the motor nerve twigs or the muscle fibres have the greater refractoriness. However, when non-propagated responses are seen at the endplate region, it is evident that some of the nerve fibres have shorter ARPs than the muscle fibres to which they are connected. In the cooled preparations it became easier to record local endplate responses, and, therefore, it appeared that the excitability of the muscle fibres had been diminished to a greater extent than that of the nerve fibres. By measuring the onsets of the local endplate responses, it was possible to show that the ARPs of the most excitable nerve twigs lengthened from $1.8 \mathrm{~ms}$ at $34^{\circ} \mathrm{C}$ to $2.9 \mathrm{~ms}$ at $28^{\circ} \mathrm{C}$ and to $5.5 \mathrm{~ms}$ at $20^{\circ} \mathrm{C}$, the $\mathrm{Q}_{10}$ being approximately $0.44\left(20^{\circ}-30^{\circ} \mathrm{C}\right)$. In order to analyse the ARPs of the muscle fibres, it was assumed that the midpoints on the recovery curves would give reasonable estimates. At temperatures of $34^{\circ}, 28^{\circ}$, and $20^{\circ} \mathrm{C}$, the corresponding ARPs were $2.3,4.5$, and $9.3 \mathrm{~ms}$ respectively; the $\mathrm{Q}_{10}$ was approximately $0.39\left(20^{\circ} \mathrm{C}-30^{\circ} \mathrm{C}\right)$.

As would be expected, impulses were conducted more slowly in the nerve fibres at the lower temperatures, causing the terminal latency to increase from $2.8 \mathrm{~ms}$ at $34^{\circ} \mathrm{C}$ to $4.8 \mathrm{~ms}$ at $20^{\circ} \mathrm{C}$. In the relatively refractory period of the nerve conduc- tion was slowed still further, with the result thap the terminal latency became as long as $8.8 \mathrm{~ms}$ a $20^{\circ} \mathrm{C}$ in one subject. In relation to aging, Tacko mann and Lehmann (1974) have shown the RRP of median nerve sensory fibres are rather larger in older subjects than in younger ones when assessed in terms of response amplitude. Although these authors were unable to demonstrate a similar difference on the basis of latency measurements, it would appear from our data that one exists, at least in the fine motor nerve branches. Unlike Farmer et al. (1960) we have not been able to demonstrate increased refractoriness of muscle fibres with aging; these authors found that ARPs were about $0.4 \mathrm{~ms}$ longer in elderly adults (6784 years) than in a younger group (15-25 years). The discrepancy between the two studies might well be attributable to the greater ages of Farmer and co-workers' elderly population, compared with those of the corresponding group in the present study (40-67 years).

It had been anticipated that increased refractoriness might be present in the distal motor nerve branches of patients with motoneurone disease and spinal muscular atrophy as part of the dyingback process but this could not be demonstrated in the four preparations studied; normal results were also obtained by Farmer et al. (1959).

In the uraemic patients it was expected that, 
despite haemodialysis, there would be sufficient changes in intracellular and extracellular electrolyte concentrations to alter membrane excitability. In line with this speculation, Tackmann et al. (1974) have found both the ARP and the RRP to be increased in sural nerve fibres. In the present study the terminal motor latency was slightly increased but it was not possible to demonstrate any difference in the refractoriness of either nerve or muscle fibres. Equally surprising has been the inability to show obvious changes in the refractoriness of muscle fibres in myotonic muscular dystrophy. In muscular dystrophies of the Duchenne, facioscapulohumeral, and limb-girdle types, Farmer et al. (1959) found that the ARPs were significantly shorter than normal, $40 \%$ of fibres having lower values than those ever encountered in normal muscle. These authors attributed their results to lowered resting potentials in dystrophic fibres but this explanation is doubtful since accommodation might be expected to occur. Further, resting depolarisation is as much a feature of myotonic dystrophy as of the other dystrophies (McComas and Mrozek, 1968). We have no convincing explanation for the finding of increased excitability in the motor nerve branches of patients with myotonic dystrophy, though it is conceivable that this abnormality is related to the neural component of the myotonic phenomenon (see Denny-Brown and Nevin, 1941).

Finally, in assessing the significance of the largely normal results in disease states, it should be noted that the results were only applicable to those nerve and muscle fibres which were excitable. No information concerning sodium channels is available for any fibres rendered inexcitable through more extensive involvement by disease. It is also possible that significant differences might have emerged between the normal and diseased populations of excitable fibres had provocative procedures, such as cooling or ischaemia, been employed.
We are indebted to Norma Zimmerman for secretarial help, and to Glenn Shine and Heidi Roth for technical assistance.

\section{References}

Buchthal, F., and Engbaek, L. (1963). Ref ractory period and conduction velocity of the striated muscle fibres. Acta Physiologica Scandinavica, 59, 199-220.

Denny-Brown, D., and Nevin, S. (1941). The phenomenon of myotonia. Brain, 64, 1-18.

Farmer, T. W., Buchthal, F., and Rosenfalck, P. (1959). Refractory and irresponsive periods of muscle in progressive muscular dystrophy and paresis due to lower motor neuron involvement. Neurology (Minneapolis), 9, 747-756.

Farmer, T. W., Buchthal, F., and Rosenfalck, P. (1960). Refractory period of human muscle after the passage of a propagated action potential. Electroencephalography and Clinical Neurophysiology, 12, 455-466.

Hodgkin, A. L., and Huxley, A. F. (1952). A quantitative description of membrane current and its application to conduction and excitation in nerve. Journal of Physiology, 117, 500-544.

Kopec, S. J., Delbeke, J., and McComas, A. J. (1978). Refractory period studies in a human neuromuscular preparation. Journal of Neurology, Neurosurgery, and Psychiatry, 41, 54-64.

McComas, A. J., and Mrozek, K. (1968). The electrical properties of muscle fibre membranes in dystrophia myotonica and myotonia congenita. Journal of Neurology, Neurosurgery, and Psychiatry, 31, 441447.

Rowland, L. P. (1976). Pathogenesis of muscular dystrophies. Archives of Neurology (Chicago), 33, 315-321.

Tackmann, W., and Lehmann, H. J. (1974). Refractory period in human sensory nerve fibres. European Neurology, 12, 277-292.

Tackmann, W., Ullerich, D., Cremer, W., and Lehmann, H. J. (1974). Nerve conduction studies cluring the relative refractory period in sural nerves of patients with uremia. European Neurology, 12, 331339. 